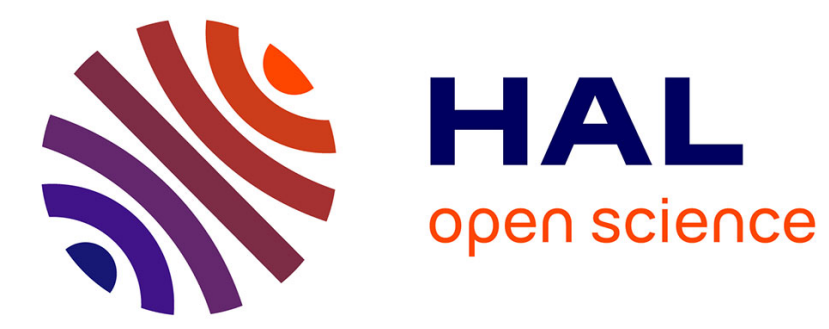

\title{
La ressource
}

Lucien Brenac

\section{To cite this version:}

Lucien Brenac. La ressource. Revue forestière française, 1976, 28 (S), pp.30-37. 10.4267/2042/21103 . hal-03396362

\section{HAL Id: hal-03396362 \\ https://hal.science/hal-03396362}

Submitted on 22 Oct 2021

HAL is a multi-disciplinary open access archive for the deposit and dissemination of scientific research documents, whether they are published or not. The documents may come from teaching and research institutions in France or abroad, or from public or private research centers.
L'archive ouverte pluridisciplinaire HAL, est destinée au dépôt et à la diffusion de documents scientifiques de niveau recherche, publiés ou non, émanant des établissements d'enseignement et de recherche français ou étrangers, des laboratoires publics ou privés. 


\section{La ressource}

\section{LA FONCTION DE PRODUCTION, CONDITION DE LA CULTURE DES BOIS}

Les usages multiples de la forêt excluent rarement la fonction de production qui, au sein de nos civilisations, est une des conditions essentielles de sa survie.

Par les caractères de leur structure et des conditions de croissance concurrentielles des arbres qui les composent —entraînant, au cours de leur longue vie liée au même sol, l'élimination du plus grand nombre - les peuplements forestiers requièrent d'ailleurs l'action de l'homme pour assurer le développement équilibré et harmonieux des sujets d'avenir tout en évitant un coûteux gaspillage de richesse et la transformation de ces forêts en cimetières d'arbres morts propices au développement de toutes sortes de nuisances (insectes, champignons).

De même, sans cette action, le passage par des phases buissonnantes ou arbustives de mortsbois, ou la substitution d'essences secondaires peu longévives aux essences nobles des plus belles futaies, ne peuvent être évités lorsque, après leur destruction par mort naturelle ou cataclysme, les peuplements anciens doivent être renouvelés.

Les interventions sylvicoles, coûteuses et délicates, ainsi nécessitées ne sont possibles que grâce à la valeur des produits de l'activité biologique de la forêt qu'elles permettent de mobiliser au profit de l'homme pour ses entreprises industrielles, artisanales ou artistiques.

Les exigences propres à la production de bois orientent, bien sûr, les opérations culturales pratiquées par le sylviculteur dans un sens favorable aux besoins de ces entreprises.

L'objectif de productivité et de rendement soutenu, par l'action sélective qu'il comporte, en même temps que la rectıerche de structures équilibrées est toutefois loin d'être, par luimême, incompatible avec la fonction d'agrément ou de protection qui, au sens large de ce terme, inclut celle-là.

\section{CARACTÉRES ORIGINAUX DE LA RESSOURCE EN MATIĖRE FORESTIĖRE}

Comparée aux autres productions de matériaux ou de matières premières, minérales ou même végétales, la production de bois présente des caractères profondément originaux.

Sans doute dans le passé et, actuellement encore, dans certains pays neufs, l'exploitation de la forêt peut-elle être, comme celle d'une carrière ou d'un gisement, exhaustive et destructive des possibilités de renouvellement.

Bien que, du fait même de l'amenuisement de la ressource, de telles conduites tendent à être très exceptionnelles - sauf dans le cas de défrichement visant à un changement radical dans l'utilisation du sol - c'est bien dans cette perspective d'irrémédiable destruction, traduite par l'utilisation abusive du terme "déboisement " que, bien souvent, réside l'origine de l'émotion ressentie par tel "écologiste » en présence des effets d'une intervention sylvicole. 
Cet amoureux de la nature n'aura pas la même réaction au vu d'un champ de blé que l'on moissonne ou d'une prairie que l'on fauche, sachant que le blé se ressème et que l'herbe repousse suivant un cycle annuel. La production forestière a pourtant en commun avec l'agricole qu'elle crée une richesse renouvelable. L'analogie ne va cependant pas beaucoup plus loin depuis que les arbres de nos forêts ne sont plus traités comme étant, avant tout, des arbres fruitiers.

La durée de croissance des essences forestières et les dimensions atteintes introduisent, en effet, des différences considérables d'échelle et une tout autre complexité. Cette complexité est, notamment, due au fait que, tout en gardant l'empreinte des saisons, la production annuelle s'intègre à la production passée par un accroissement anatomiquement distinct mais néanmoins inséparable du matériel accumulé qui lui sert de support.

La récolte est dès lors distincte de la production annuelle : elle ne porte pas directement sur celle-ci mais sur un certain nombre de tiges du peuplement.

Il n'y a pas seulement récolte mais opération de sélection. De plus la coupe, par son importance et les choix réalisés, conduit à l'augmentation, ou à la réduction, du matériel sur pied comme elle a effet sur la structure des classes d'âge ou de dimensions. Par cette double action le prélèvement agit sur la production en modifiant l'utilisation de la capacité biologique de la station qui varie avec la composition, la structure, la consistance et l'âge du peuplement.

La comparaison parfois tentée entre la sylviculture et l'élevage peut trouver une certaine justification dans les constatations faites ci-dessus. Outre celle résultant de la durée de vie, une différence radicale réside pourtant dans la non extensivité de la surface offerte aux peuplements qui provoque l'intensité de la concurrence vitale entraînant, à défaut d'intervention. une sévère élimination par voie de sélection naturelle $\left({ }^{1}\right)$.

Une autre différence marquée avec la production agricole, découlant des caractères originaux ci-dessus analysés de la culture forestière, est que le moment de la récolte n'est pas naturellement déterminé et, qu'en outre, cette récolte est presque toujours hétérogène quant aux catégories d'utilisation qu'elle renferme, lesquelles varient avec la structure, l'âge du peuplement et la nature de l'opération sylvicole. Ces catégories d'utilisation ne sont d'ailleurs pas entièrement prédéterminées : telle tige, partie de tige ou branche pouvant être apte à plusieurs usages.

Est donc particulièrement importante la place laissée aux choix à réaliser dans la fixation de la nature, de l'assiette et de la quotité des coupes qui, à partir de la connaissance de l'état actuel des peuplements, auront une influence déterminante sur le niveau et la nature de la production. Il en est de même après exploitation quant au tri et au débit des bois.

\section{LA CONNAISSANCE DE L'ÉTAT DE LA FORÊT FRANÇAISE — L'INVENTAIRE FORESTIER NATIONAL}

Jusqu'à une date récente, ne remontant guère au-delà d'une ou deux décennies, la connaissance de la ressource en matière forestière était très imparfaite, pour ne pas dire presque inexistante. Sans doute était-il possible, pour les forêts soumises au régime forestier — à défaut des données des aménagements trop partielles et pas aisément cumulables - d'utiliser les statistiques annuelles de produits vendus et délivrés comme fournissant une limite infé-

(1) La traditionnelle et très ancienne liaison entre les eaux et les forêts _ indéracinable de la conscience populaire - peut trouver une de ses justifications dans l'analogie plus grande encore avec la vie de la forêt que peut offrir le milieu aquatique ou. avec une semblable intensité de la concurrence vitale et parfois, une aussi grande longévité, se manifeste une croissance gardant la marque du temps (écailles des poissons, coquilles des lamellibranches) à ce point comparable qu'elle peut être justiciable de la même formulation mathématique (la formule de von Bertallanffy (1951) $\frac{\mathrm{dV}}{\mathrm{dt}}=\mathrm{a} \mathrm{V}^{2 / 3}-\mathrm{bV}$ relative a la croissance des arbres avait déjà été indiquée par Kostitzin, en 1937, comme pouvant traduire la croissance du poids de la brème). 


\section{BRENAC}

rieure de la possibilité de ces forêts sans pouvoir toutefois être en mesure d'estimer l'écart existant avec la ressource réellement disponible.

Pour les forêts privées, représentant au moins les deux tiers de la surface boisée, les informations fournies par l'enquête Daubrée (1904-1908) sur les superficies boisées et sur les volumes exploités - quoiqu'étant encore assez souvent utilisées — étaient, en tout état de cause, périmées et malgré son indéniable utilité, l'enquête sür la production de la branche exploitation forestière ne pouvait donner que l'évaluation de la partie principale de la coupe annuelle, englobant d'ailleurs l'ensemble des formations boisées de toutes catégories de propriété.

En ce qui concerne les seules superficies on peut estimer que la situation au 1 er janvier 1958, figurant à l'Annuaire des Eaux et Forêts de 1959, des "superficies des bois et terrains soumis au régime forestier et des forêts particulières » présentait, sans doute, avec un total de 11630978,69 ha, un écart de l'ordre de 2 millions d'hectares avec la réalité (d'autant plus que cette superficie n'incluait pas moins de 450000 ha de terrains non boisés).

Avec la nécessité de répondre aux besoins accrus des industries du bois et le souci d'organisation de la forêt privée, qui devait aboutir à la loi du 6 août 1963, l'insuffisance de nos connaissances, plus fortement ressentie, a conduit à l'ordonnance no 58-880 du 24 septembre 1958.

Cette ordonnance, devenue l'article 204-1 du Code forestier, a confié au ministre de l'Agriculture l'exécution de l'inventaire permanent des ressources forestières nationales, après qu'il eut été question de réaliser un «recensement forestier " à l'occasion du recensement général de l'agriculture de $1955\left(^{2}\right)$.

Si cette décision a pu paraître tardive, confrontée à l'exemple de nombreux pays, elle a par contre, conduit à la fixation d'objectifs plus ambitieux que ceux généralement visés. La mission confiée aux ingénieurs chargés de la réalisation de cet inventaire a été, en effet, comprise dans le sens le plus large quant aux formations à inventorier puisqu'elle concerne, non seulement les forêts, mais aussi les peupleraies, les plantations d'alignement, les haies, les arbres forestiers épars ainsi que les landes et autres vacants.

L'inventaire, réalisé par départements et régions forestières, doit recueillir des données aussi complètes que possible, susceptibles de caractériser les formations ci-dessus au point de vue statistique et dynamique, qui concernent les superficies, les volumes sur pied et exploités, les accroissements (diamètre, hauteur, volume) et les nombres d'arbres selon l'usage du sol, les grandes catégories de propriété (domanial, autres terrains soumis au régime forestier, particulier), les types de peuplements, les essences, les traitements sylvicoles, les classes $d^{\prime}$ âge et de diamètre.

Ėtant donné la diversité de ses objectifs qui s'appliquent à des formations compactes comme les forêts, linéaires comme les haies et les plantations d'alignement, claires ou diffuses comme les arbres épars, la méthode utilisée, qui trouve son unité dans la mise en œuvre de l'échantillonnage statistique, a dû être adaptée à la diversité de ces formations.

A la différence des enquêtes agricoles par sondages essentiellement fondées sur des interrogations par questionnaires $\left({ }^{3}\right)$, ce qui les expose à toutes les imperfections et déformations éventuelles d'une connaissance subjective, l'Inventaire forestier a été fondé sur des données originales provenant uniquement de mensurations prises sur le terrain après analyse sur photographies aériennes des formations à inventorier.

(2) Ce projet n'a pas eu de suite en raison, d'une part, d'idées préconçues tirées de l'expérience des sondages en matière agricole, telles que l'assimilation du massif forestier à l'exploitation agricole en tant qu'unité statistique de sondage, mais aussi par manque de moyens financiers dont devait également pâtir l'Inventaire forestier à ses débuts.

(3) "Parmi les enquêtes statistiques agricoles réalisées par le Service Central des enquêtes et ètudes statistiques du ministère de l'Agriculture, l'enquête Utilisation du territoire est la seule á présenter la particularité d'être basée sur le principe d'une observation directe donc objective du territoire..." (Coliections de Statistique agricole, Étude n 124, mai 1974). Ayant abandonné l'utilisation des plans cadastraux au bénéfice des photographies aériennes sondées par grilles de points à l'exemple de l'Inventaire forestier, cette enquête ne procède cependant à aucune prise de mesures sur le terrain. 
Il a pu être question de période pré-scientifique, puis de période à tendance scientifique à partir de 1960, pour caractériser les méthodes appliquées dans les enquêtes agricoles $\left({ }^{4}\right)$. Si on englobe l'Inventaire forestier dans les "enquêtes", ce qui semble admis en raison de la généralité de ce vocable, il convient de ne pas se laisser abuser par son acception la plus courante qui évoque le recueil d’informations, non pas directement sur l'objet de l'enquête. mais auprès des personnes par déclarations individuelles, questionnaires ou évaluations par des commissions communales. Si la "statistique forestière de 1878 " et "l'Enquête Daubrée » (1904-1908) appartiennent à cette dernière catégorie et peuvent être rattachées à la période pré-scientifique des enquêtes agricoles, par contre les méthodes d'échantillonnage statistique probabiliste et les mensurations précises effectuées sur le terrain pour l'Inventaire forestier national lui ont conféré, dès qu'il a été entrepris en 1960, un caractère qui participe pleinement de l'objectivité scientifique.

Il est vrai, qu'en raison de l'originalité et de la complexité de la production forestière ci-dessus soulignées, des données complètes, définissant l'état des peuplements et ayant la valeur que leur confère ce caractère scientifique, sont indispensables pour aménager à court et à long terme l'utilisation de la ressource.

(4) La Statistique agricole française. Étude $n^{\circ} 140$.

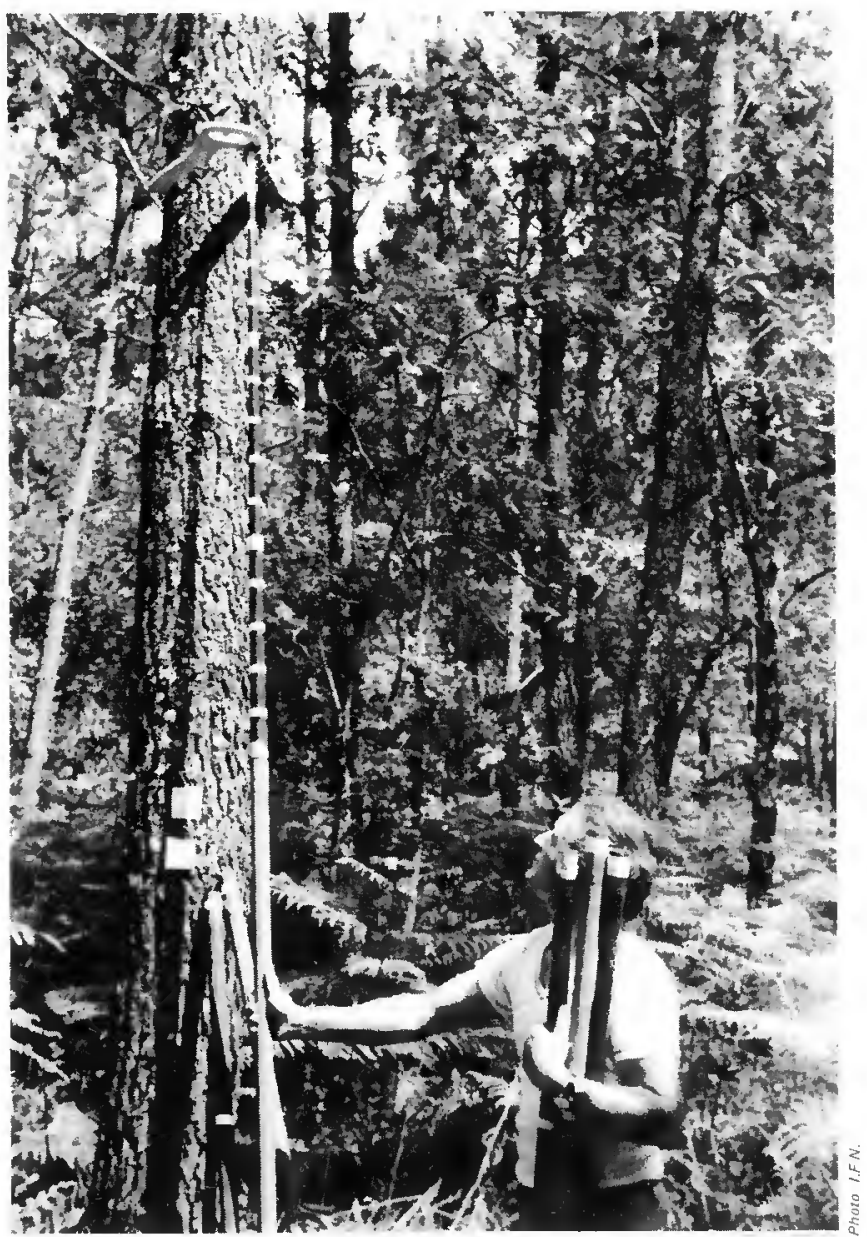




\section{ÉTAT D'AVANCEMENT DE L'INVENTAIRE FORESTIER NATIONAL}

La mobilisation des moyens en personnels et crédits indispensables pour la réalisation de l'inventaire ayant été très progressive $\left({ }^{5}\right)$, jusqu'à la mise en place complète des cinq échelons interrégionaux qui se partagent le territoire et du Centre de calcul chargé de l'exploitation informatique des données brutes de l'inventaire, le rythme d'avancement souhaité pour réaliser un cycle complet d'inventaire en 10 ans n'a été atteint qu'à partir de 1973 mais est régulièrement soutenu depuis avec 9 départements par an (sur 89 au total, la région parisienne et la Corse étant chacune comptées pour 2 départements, le Territoire-de-Belfort étant joint à la Haute-Saône).

Dans ces conditions l'état d'avancement des travaux d'inventaire était le suivant à la date du 1 er mai 1977 :

\section{1 er cycle d'inventaire}

- 74 départements pour lesquels les travaux d'inventaires sont terminés sur le terrain dont :

- 59 ayant fait l'objet de publications

- 5 exploités dont les résultats sont disponibles

- 64 départements au total, ayant :

$\begin{array}{lll}\text { - superficie territoriale } & \text { de } 40515640 \text { ha }(73,8 \%) \\ \text { - superficie boisée } & \text { de } 10267851 \text { ha }(25,3 \%) \\ \text { - superficie landes } & \text { de } 2823455 \text { ha }(6,9 \%) \\ \text { - superficie peupleraies } & \text { de } & 69100 \text { ha } \\ \text { - éléments linéaires } & & 1150000 \mathrm{~km}\end{array}$

- 10 départements en attente d'exploitation

- 9 départements en cours d'inventaire

- 6 départements restant à inventorier

$2^{e}$ cycle d'inventaire

- 1 département terminé sur le terrain

- 5 départements en cours

-2 sur le terrain

- 3 sur couverture photographique

\section{DONNÉES DE L'INVENTAIRE FORESTIER NATIONAL - EXTRAPOLATION A LA FRANCE ENTIĖRE}

II a paru possible, à partir des données disponibles pour 64 départements, d'étendre les résultats à l'ensemble du territoire. L'utilisation d'autres statistiques départementales (enquête sur la branche d'activité "exploitations forestières », enquête " utilisation du Territoire », statistiques de l'Office national des forêts) dont certaines données, pas nécessairement de même

(5) II avait été prévu que, moyennant la création d'emplois budgétaires (Rapport Forêt pour le lile Plan), les personnels nécessaires à la réalisation de l'inventaire forestier seraient fournis par l'Administration des eaux et forêts. Le montant - très réduit de ce fait - et l'affectation budgètaire des crédits avaient été fixés d'après cette prévision, qui ne fut pas réalisée, tout au moins en ce qui concerne les personnels d'exécution. N'ont également pas reçu d'application les prévisions, résultant des termes même de la loi, relatives à "l'aide technique de l'Institut national de la statistique et des études économiques " qui devait, notamment, comporter le traitement informatique des données. 


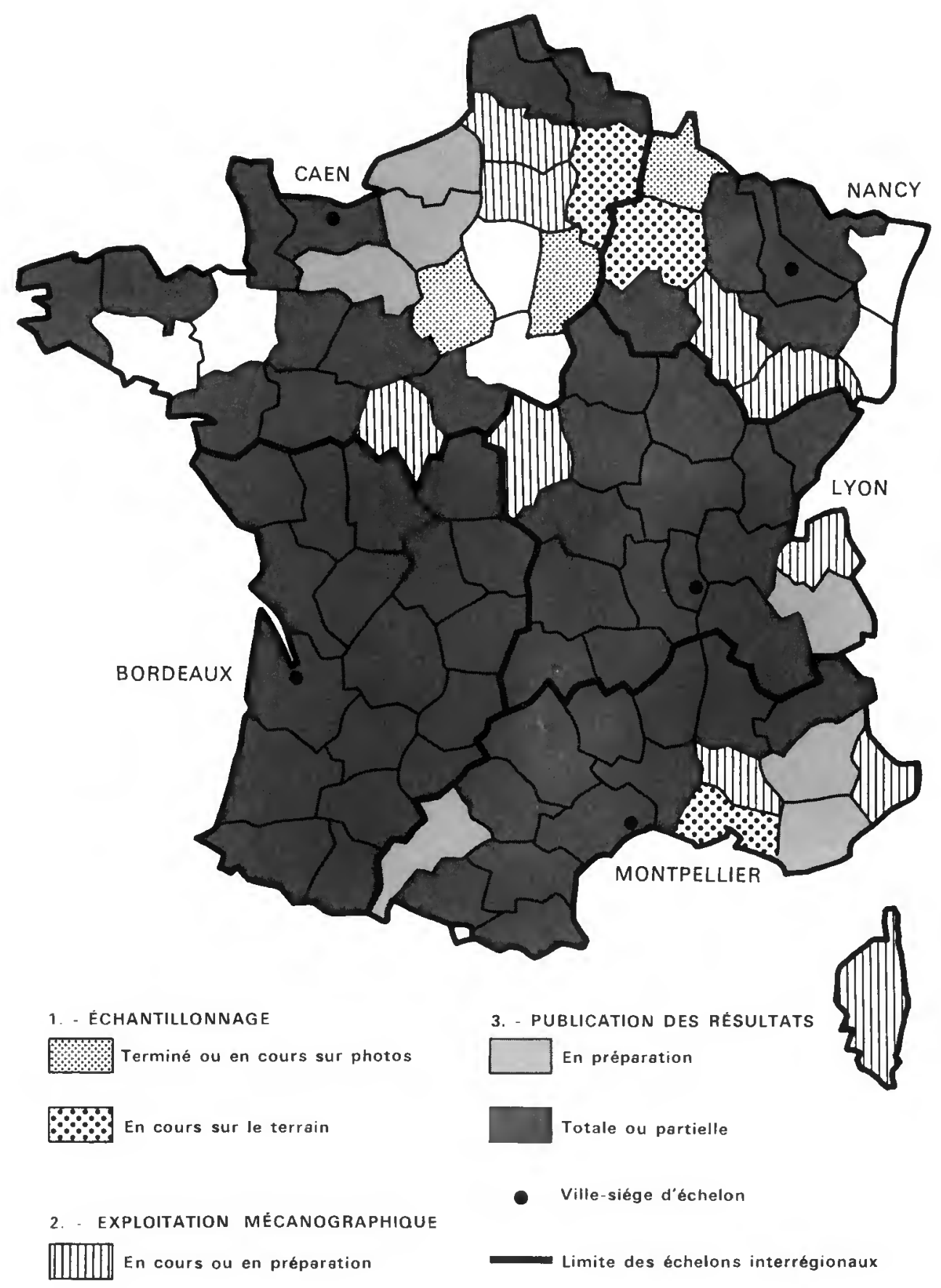


nature que celles de l'inventaire, mais, néanmoins assez étroitement corrélées avec elles, a permis, en leur faisant jouer le rôle de variables explicatives dans un ajustement par régression, de réaliser ces extrapolations dont les résultats figurent ci-dessous :

- Superficie totale des bois et forêts (peupleraies exclues) : 13480000 ha

- Forêts de production

12320000 ha

- Boqueteaux et bosquets $(0,05$ à $4 \mathrm{ha})$ :

680000 ha

- Forêts de protection :

480000 ha

- Forêts soumises au régime forestier $\left(^{6}\right)$ : 3600000 ha

- Forêts de l'État :

1360000 ha

- Forêts des communes et autres forêts :

2240000 ha

- Formations boisées hors forêt :

- Peupleraies en plein :

160000 ha

- Haies :

$1500000 \mathrm{~km}$

- Alignements :

$93000 \mathrm{~km}$

- Volumes contenus dans les haies, alignements et arbres forestiers épars :

73 millions $\mathrm{m}^{3}$

- Forêts de production et petits massifs

(boqueteaux et bosquets) :

13000000 ha

- Composition :

- feuillus :

8770000 ha

- résineux :

4230000 ha

- Volumes sur pied : 1360 millions $\mathrm{m}^{3}$

- feuillus :

800 millions $\mathrm{m}^{3}$

- résineux :

560 millions $\mathrm{m}^{3}$

- Accroissements : 46,4 millions $\mathrm{m}^{3}$

- feuillus:

25,6 millions $\mathrm{m}^{3}$

- résineux :

20,8 millions $\mathrm{m}^{3}$

- Accroissements par catégories de propriété :

- domanial :

5,5 millions $\mathrm{m}^{3}$

- communal et autres forêts soumises :

6,5 millions $\mathrm{m}^{3}$

- particulier :

34,4 millions $\mathrm{m}^{3}$

Les données de base se rapportant à des inventaires départementaux échelonnés dans le temps - 54 d'entre eux datant de moins de 10 ans et 10 de plus de dix ans - les résultats obtenus ne peuvent être rapportés à une date précise, ayant plutôt le caractère de moyennes mobiles auxquelles on peut, toutefois, accorder une assez grande stabilité au cours de la période en cause quant aux ordres de grandeur que ces résultats prétendent seuls traduire. Les inventaires départementaux fournissent des données beaucoup plus complètes et détaillées à l'intérieur des différents domaines d'étude que sont, outre les départements et les grandes

(6) II ne s’agit, évidemment, que des surfaces boisées. 
catégories de propriétés, les régions forestières et les types de peuplement. Ces résultats ne sont toutefois pas suffisants pour déterminer la ressource annuellement mobilisable, celle-ci dépendant aussi, en effet, de la façon dont seront conduites les exploitations qui devront maintenir, ou accroître, la capacité de production tout en permettant à la forêt de continuer à remplir les autres fonctions d'intérêt général qui lui sont dévolues.

La ressource mobilisable peut être, dans ces conditions, inférieure, égale ou supérieure à la production nette des peuplements suivant le degré d'équilibre qu'ils présentent quant à la répartition des classes d'âge ou de dimensions.

Il est, d'autre part, assez évident qu'il pourra exister un écart important entre la ressource physiquement mobilisable et les produits réellement exploités au cours d'une période donnée, ceux-ci dépendant d'un grand nombre de facteurs externes supplémentaires qui ne sont pas seulement de nature économique, en raison des effets de structures foncières morcellées et incertaines telles que les expressions "sylviculture appliquée », "intention du propriétaire " sont dans de trop nombreux cas à peu près vides de sens.

Il est possible, non d'analyser et de quantifier un tel "système " des plus complexes qui résiste à la démarche décomposante et hiérarchique du traitement cartésien, mais, sans doute, d'en suivre, tout au moins, les effets dans leur résultante grâce au renouvellement périodique de l'inventaire et d'autres enquêtes (enquête de branche, enquête sur la structure de la forêt privée) qui doivent permettre de dégager les tendances et, dans une certaine mesure, d'en prévoir l'évolution.

Lucien BRENAC

Ingénieur général du G.R.E.F. Chef du Service

INVENTAIRE FORESTIER NATIONAL

1 ter, avenue de Lowendal 75700 PARIS 